\title{
Morbidity after Hemorrhage in Children with Untreated Brain Arteriovenous Malformation
}

\author{
Li Ma ${ }^{a-c, g}$ Helen Kim ${ }^{g, h}$ Xiao-Lin Chen ${ }^{a, b, g}$ Chun-Xue Wu ${ }^{d}$ Jun Ma ${ }^{d}$ \\ Hua Su ${ }^{g}$ Yuanli Zhao a-c, e,f \\ ${ }^{a}$ Department of Neurosurgery, Beijing Tiantan Hospital, Capital Medical University, ${ }^{b}$ China National Clinical \\ Research Center for Neurological Diseases, 'Department of Neurosurgery, Peking University International Hospital, \\ Peking University Health Science Center, d Department of Neuroradiology, Beijing Tiantan Hospital, Capital Medical \\ University, ${ }^{e}$ Center of Stroke, Beijing Institute for Brain Disorders, and ${ }^{\mathrm{f} B e i j i n g ~ K e y ~ L a b o r a t o r y ~ o f ~ T r a n s l a t i o n a l ~}$ \\ Medicine for Cerebrovascular Disease, Beijing, PR China; ${ }^{9}$ Center for Cerebrovascular Research, Department of

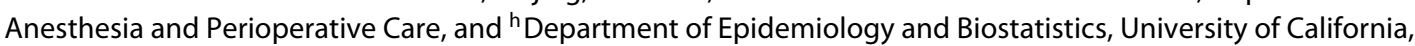 \\ San Francisco, CA, USA
}

\section{Key Words}

Arteriovenous malformation · Child · Morbidity · Risk assessment

\begin{abstract}
Background: Children with untreated brain arteriovenous malformations (bAVM) are at risk of encountering life-threatening hemorrhage very early in their lives. The primary aim of invasive treatment is to reduce unfavorable outcome associated with a bAVM rupture. A better understanding of the morbidity of bAVM hemorrhage might be helpful for weighing the risks of untreated bAVM and invasive treatment. Our aim was to assess the clinical outcome after bAVM rupture and identify features to predict severe hemorrhage in children. Methods: We identified all consecutive children admitted to our institution for bAVMs between July 2009 and December 2014. Clinical outcome after hemorrhagic presentation and subsequent hemorrhage was evaluated using the modified Rankin Scale (mRS) for children. The association of demographic characteristics and bAVM morphology with severe hemorrhage ( $\mathrm{mRS}>3$ or requiring emergency hema-
\end{abstract}

toma evacuation) was studied using univariate and multivariable regression analyses. A nomogram based on multivariable analysis was formulated to predict severe hemorrhage risk for individual patients. Results: A total of 134 patients were identified with a mean treatment-free followup period of 2.1 years. bAVM ruptured in 83 (62\%) children: 82 had a hemorrhage at presentation and 6 of them experienced a recurrent hemorrhage during follow-up; 1 patient had other diagnostic symptoms but bled during follow-up. Among them, 49\% (41/83) had a severe hemorrhage; emergency hematoma evacuation was required in $28 \%$ of them $(23 / 83)$, and $24 \%(20 / 83)$ remained as disabled (mRS $\geq 3)$ at last follow-up. Forty-six percent (38/82) of children with hemorrhagic presentation were severely disabled ( $m R S>3$ ). Forty-three percent (3/7) were severely disabled after subsequent hemorrhage. The annual rate of severe subsequent hemorrhage was $1 \%$ in the overall cohort and 3.3\% in children with ruptured presentation. All the subsequent severe hemorrhage events occurred in children with severe hemorrhage history $(7 \%, 3 / 41)$. Periventricular location, non-temporal lobe location, and long draining vein were predictors for severe hemorrhage in pediatric untreated bAVMs. A no-

\section{KARGER}

(c) 2017 S. Karger AG, Basel

E-Mail karger@karger.com

www.karger.com/ced
Yuanli Zhao, MD

Department of Neurosurgery, Beijing Tiantan Hospital

Capital Medical University

No. 6 Tiantan Xili, Dongcheng District, Beijing 100050 (PR China)

E-Mail zhaoyuanli@126.com 
mogram based on bAVM morphology was contracted to predict severe hemorrhage risk for individual patients, which was well calibrated and had a good discriminative ability (adjusted C-statistic, 0.72). Conclusions: Evaluating bAVM morbidity and morphology might be helpful for weighing the risks of untreated bAVM in pediatric patients.

(c) 2017 S. Karger AG, Basel

\section{Introduction}

Children with untreated brain arteriovenous malformations (bAVM) are at risk of experiencing life-threatening hemorrhage [1]. The primary aim of invasive treatment is to reduce unfavorable outcomes associated with bAVM rupture [2]. Recent studies suggest a lower morbidity of bAVM rupture than intracranial hemorrhage resulting due to other causes $[3,4]$. Therefore, it might be more helpful for clinical decision-making if we can determine the morbidity of an untreated bAVM. However, most pediatric bAVMs present with hemorrhage, and conservative follow-up methods of ruptured bAVMs are scarce, making it difficult to assess long-term outcome in the natural course $[1,5]$. Additionally, treatment-free time after hemorrhage varies between individuals, further hindering the comparison of clinical outcome. A recent study indicated that although intervention would change bAVM prognosis, the long-term outcome was still correlated with neurological function at presentation [6]. In addition, neurological evaluation immediately after the index event was more likely to reveal the actual impact of hemorrhage after bAVM rupture [3]. Therefore, an alternative approach to study the morbidity of bAVM might be to assess the immediate post-hemorrhage neurological function, which is the functional outcome at the same time point and it correlated with long-term prognosis.

A better understanding of the morbidity of bAVMs will also contribute to risk stratification of functional outcome. Previous reports identified posterior fossa, eloquent location, and associated aneurysm as potential predictors for severe hemorrhagic presentation in ruptured bAVM [7]. Our recent study in pediatric patients suggested a trend toward poorer neurological function at presentation and more emergency intervention in periventricular bAVM [8]. In this study with follow-up data before treatment, we aimed to (1) assess the neurological function after hemorrhagic presentation and subsequent hemorrhage and at follow-up in children with untreated bAVMs; (2) explore predictive features of severe hemorrhage in pediatric $\mathrm{bAVM}$.

\section{Materials and Methods}

The study protocol was approved by the Institutional Review Board of our institution. Written informed consent was obtained from all participants and their guardians at the time of admission.

\section{Patients and Study Design}

The AVM database at our institution is a prospectively maintained database collecting demographic, clinical, and neuroradiological data for all patients with a confirmed angiographic or histological diagnosis of intracranial AVM treated at our institute [8]. This database was reviewed to identify all consecutive children diagnosed with bAVMs between July 2009 and December 2014. Pediatric patients were defined as patients who were 18 years of age or younger when angiographic diagnosis of bAVM (excluding dural and pial arteriovenous fistulas and vein of Galen malformations) was made for the first time.

bAVM hemorrhage was defined as a symptomatic clinical event with signs of fresh intracranial blood on CT or MRI and/or in the CSF with no easily identifiable alternative source that was more likely than the bAVM to be the cause. bAVM initial presentation and follow-up data before lesion-targeted treatment were evaluated. Neurological function was assessed using modified Rankin Scale (mRS) for children with age-specific modification as described previously (online suppl. Methods; for all online suppl. material, see www.karger.com/doi/10.1159/000458731) [9]. mRS scores were recorded within $24 \mathrm{~h}$ (1) after admission, (2) after subsequent hemorrhage, and (3) at follow-up before bAVM treatment [10]. A clinician who was not directly involved in the care of these patients performed all scale assessments. Considering the impact of varied follow-up time between presentation and treatment on neurological function, bAVM severe hemorrhage was defined as (1) mRS score $>3$ or (2) requiring emergency hematoma evacuation after hemorrhagic presentation or subsequent hemorrhage. Emergency hematoma evacuation was considered in patients with GCS $\leq 8$ or large hematoma with midline shift. The annual hemorrhage/severe hemorrhage rate was calculated as the number of hemorrhages/severe hemorrhages divided by the total patient-years of treatment-free follow-up. For patients with conservative observation, this follow-up period was the interval between diagnosis and last follow-up. For patients who underwent interventional treatment, this period was from the time the diagnosis was made to the time of lesion-targeted treatment.

\section{Neuroradiological Review}

$\mathrm{MR}, \mathrm{CT}$, and angiographic images were evaluated by consensus between 2 experienced neuroradiologists (J.M. and Gu Wei-Bin) who were blinded to the clinical information. A structured list of angiographic and MR features (location, size, venous drainage, and arterial supply) was retrospectively scored using a protocol that conformed to the consensus of bAVM research-reporting terminology recommended by a Joint Group [11].

bAVM location was described as positive for frontal, temporal, parietal, occipital, basal ganglia, thalamus, internal capsule, corpus callosum, brain stem, and cerebellum, if bAVM nidus involved the aforementioned regions. Superatentorial bAVMs were further dichotomized as deep (basal ganglia, thalamus, internal capsule, and corpus callosum) and superficial (all other locations) [12]. A posterior fossa location was defined as brainstem, cerebellum, or both. Eloquent brain was defined as previously reported in the SpetzlerMartin grading system. bAVMs were also classified as periven- 


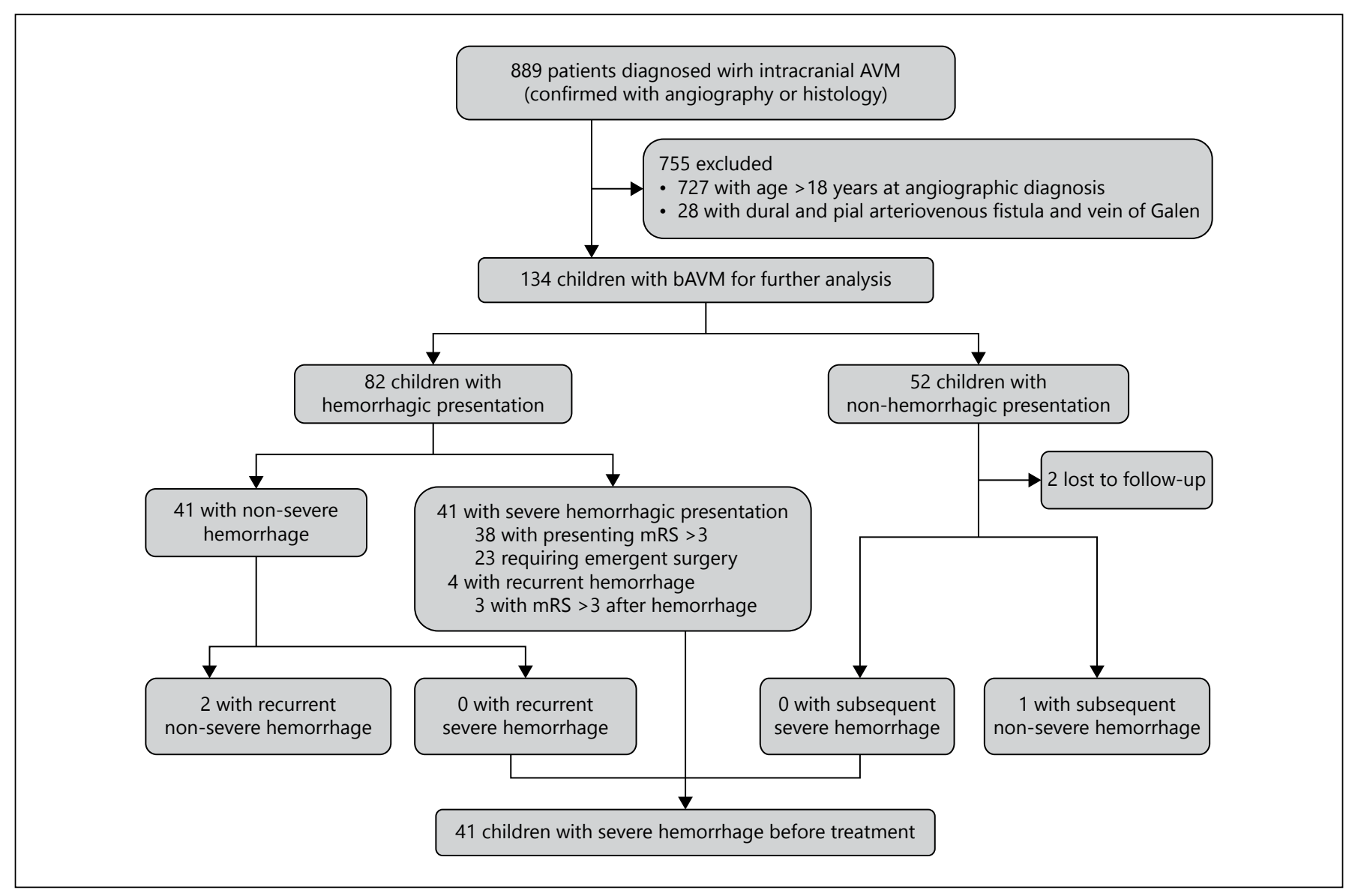

Fig. 1. Flow diagram of the study population.

tricular location if the nidus (with a contrast-enhancement or flow void) contacted the ependymal lining of the ventricle on contrastenhanced T1- and T2-weighted images $[8,13]$.

Venous drainage was dichotomized into exclusively deep venous drainage or non-exclusively deep venous drainage (superficial-only drainage or superficial and deep drainage). Long draining vein was defined as a draining vein with a length longer than $3 \mathrm{~cm} \mathrm{[14].} \mathrm{An} \mathrm{aneurysm} \mathrm{was} \mathrm{defined} \mathrm{as} \mathrm{a} \mathrm{saccular} \mathrm{dilation} \mathrm{of} \mathrm{the}$ lumen more than 2 times the width of the arterial vessel that carried the dilation [15]. Associated aneurysms only included aneurysms related to shunt flow [16].

Each nidus was measured in 3 dimensions on the latest contrast-enhanced MRI and angiogram before bAVM rupture. The largest diameter (in millimeters) among the 3 dimensions was recorded as the maximal AVM size for further analysis.

\section{Statistical Analysis}

Data were analyzed using IBM SPSS Statistics version 22.0 (IBM, Armonk, NY, USA) and R software (version 3.3.1). Statistical significance was set at $p<0.05$. For neuroradiological and clinical data, patients with and without severe hemorrhage were compared using descriptive statistics, including $t$ test or Wilcoxon rank-sum test for continuous variables and $\chi^{2}$ test or Fisher's exact test for categorical variables.

Severe Hemorrhage Risk in Children with Untreated bAVM
Both univariate and multivariable logistic regression were performed to estimate the risk of severe hemorrhage. We used 2 multivariable models to test the potential predictors that have been reported to be predictive of hemorrhage as listed in tables. Potential multi-collinearity and model fit were evaluated (online suppl. material). With the multivariable analysis, we formulated a nomogram to predict severe hemorrhage risk. Nomogram performance was assessed using the calibration curve and C-statistic to measure internal calibration and discriminative ability. C-statistic over 0.7 indicates a good model.

\section{Results}

\section{Study Population}

A total of 134 pediatric patients with bAVM were identified from among 889 patients with intracranial AVM (Fig. 1; Table 1). The age at diagnosis ranged from 1 to 18 years (mean age $12 \pm 3.9$ years). None of the patients had familial bAVM or hereditary hemorrhagic telangiectasia. Ninety-four percent of bAVMs in this pediatric cohort were supratentorial. Thirty-seven percent 
Table 1. Characteristics of 134 pediatric arteriovenous malformation patients with severe hemorrhage at presentation or during treatment-free follow-up*

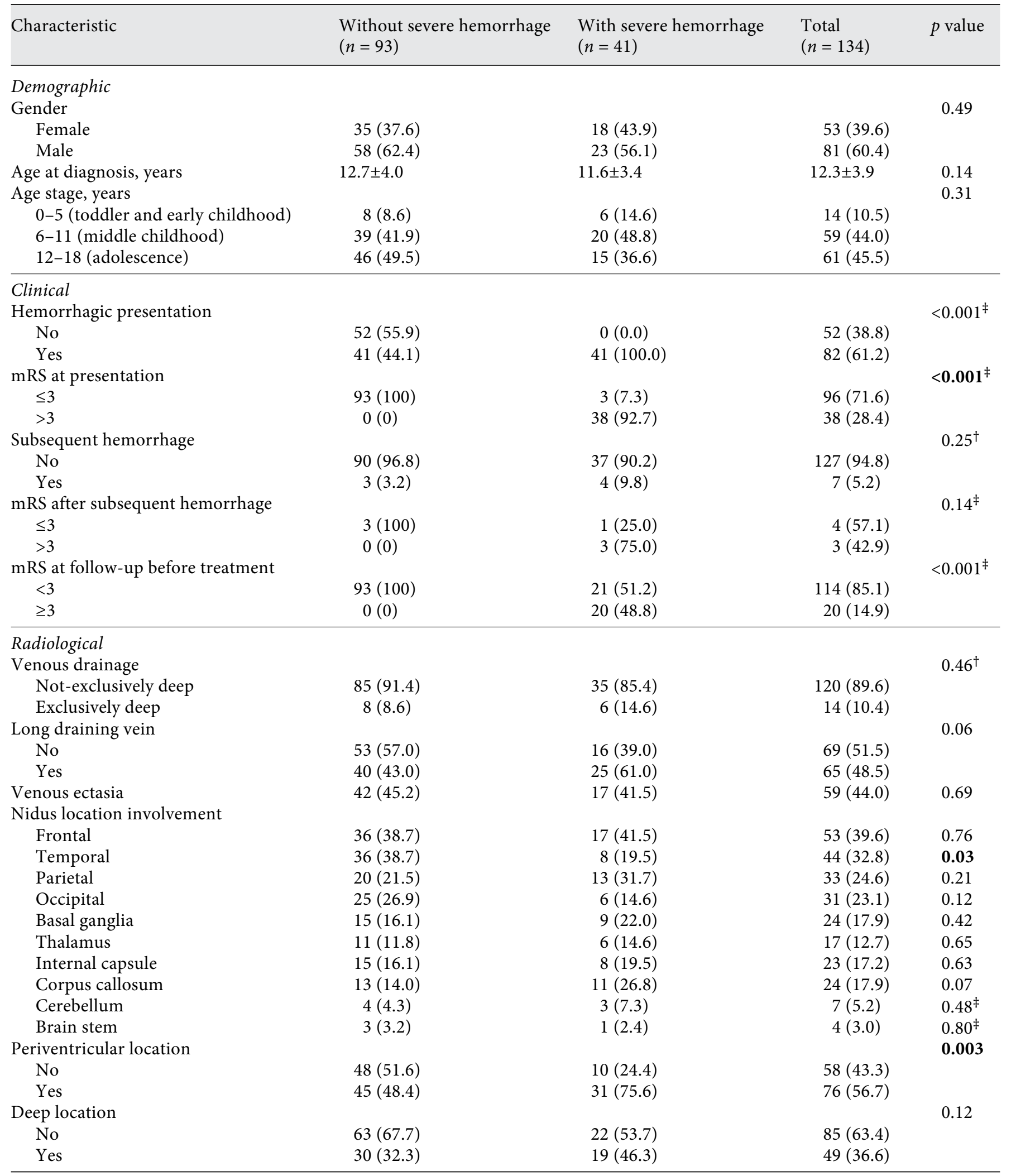


Table 1. (continued)

\begin{tabular}{|c|c|c|c|c|}
\hline Characteristic & $\begin{array}{l}\text { Without severe hemorrhage } \\
(n=93)\end{array}$ & $\begin{array}{l}\text { With severe hemorrhage } \\
(n=41)\end{array}$ & $\begin{array}{l}\text { Total } \\
(n=134)\end{array}$ & $p$ value \\
\hline \multicolumn{4}{|l|}{ Posterior fossa location } & $0.97^{\dagger}$ \\
\hline Yes & $5(5.4)$ & $3(7.3)$ & $8(6.0)$ & \\
\hline \multicolumn{4}{|l|}{ Eloquence } & 0.63 \\
\hline \multicolumn{4}{|l|}{ Associated aneurysm } & 0.66 \\
\hline No & $84(90.3)$ & $36(87.8)$ & $120(89.6)$ & \\
\hline Yes & $9(9.7)$ & $5(12.2)$ & $14(10.4)$ & \\
\hline Maximal nidus size, $\mathrm{mm}$ & $11.5 \pm 4.2$ & $39.1 \pm 20.0$ & $39.5 \pm 18.7$ & 0.88 \\
\hline \multicolumn{4}{|l|}{ Spetzler-Martin grade } & $0.83^{\S}$ \\
\hline I-II & $50(53.8)$ & $22(53.7)$ & $72(53.7)$ & \\
\hline Mean, years & 2.51 & 1.30 & 2.14 & \\
\hline \multicolumn{5}{|c|}{$\begin{array}{l}* \text { Table entries are } n(\%) \text { or mean } \pm \text { SD. } p \text { value in boldface indicates statistical significance. } \\
{ }^{\dagger} p \text { values are from the } \chi^{2} \text { test (correction for continuity). } \\
\ddagger p \text { values are from the Fisher's exact test. } \\
\S p \text { values are from the Mann-Whitney } U \text { test. }\end{array}$} \\
\hline
\end{tabular}

were deep location and $47 \%$ involved the eloquent brain. Exclusively deep venous drainage was found in $10 \%$ of the bAVMs and long draining vein in $49 \%$. Associated aneurysm was found in $10 \%$ of the children. Fifty-four percent of the bAVMs were Speztler-Martin grade I-II, $30 \%$ were grade III, and $16 \%$ were grade IV-V. Sixteen percent of cases were followed without interventional treatment.

\section{Clinical Outcome after bAVM Hemorrhage and at Follow-Up before bAVM Treatment}

In 82 children $(82 / 134,61 \%)$ with hemorrhage as initial presentation, the median delay between AVM rupture and initial neurological assessment was 1.5 days (mean 8 days). Among them, 46\% (38/82) had an immediate post-hemorrhage mRS $>3$ and $28 \%$ of them $(23 / 82)$ underwent emergency hematoma evacuation. A total of 41 patients $(41 / 134,31 \%)$ had a hemorrhagic presentation with severe neurological deficit $(m R S>3)$ and/or requiring emergency intervention (Fig. 1, 2; Table 2).

Complete follow-up was obtained for all but 2 (1.5\%) patients (Fig. 1). During a total follow-up period of 286.9 person-years between presentation and treatment (median 2.3 months, mean 2.1 years), 8 subsequent AVM hemorrhage events occurred in 7 patients, yielding an annual subsequent hemorrhage rate of $2.8 \%$. One subsequent hemorrhage occurred in a child who had non-hemorrhagic presentation (annual rupture rate of $0.5 \%$ during 198.4 patient-years follow-up) and 7 hemorrhages reoccurred in 6 children with previous hemorrhagic presentation (annual re-bleeding rate of $7.9 \%$ during 88.5 patient-years follow-up). In children with conservative management, one subsequent hemorrhage occurred in a child after hemorrhagic presentation (annual rupture rate of $0.5 \%$; Table 2 ).

Most children had a recurrent hemorrhage between the age of 8 and 13 years (mean age $10 \pm 3.9$ years, interquartile range [IQR] 8-13 years), and the median interval between 2 hemorrhagic events was 1.77 years (mean $2.6 \pm$ 3.2 years, IQR 12 days -7 years). Among them, 3 children $(3 / 7,43 \%)$ had an immediate mRS $>3$ after bAVM rupture, who all had hemorrhagic presentation with $\mathrm{mRS}>3$ (Fig. 2). No emergency hematoma evacuation was initiated and no child died after subsequent bleeding. The annual rate of severe subsequent hemorrhage was $1 \%$ in this pediatric cohort with bAVM, and 3.3\% in children with ruptured presentation (Table 2). All the subsequent se- 
Fig. 2. $m R S$ for children with bAVM of different age stages after bAVM hemorrhage and at last treatment-free follow-up.

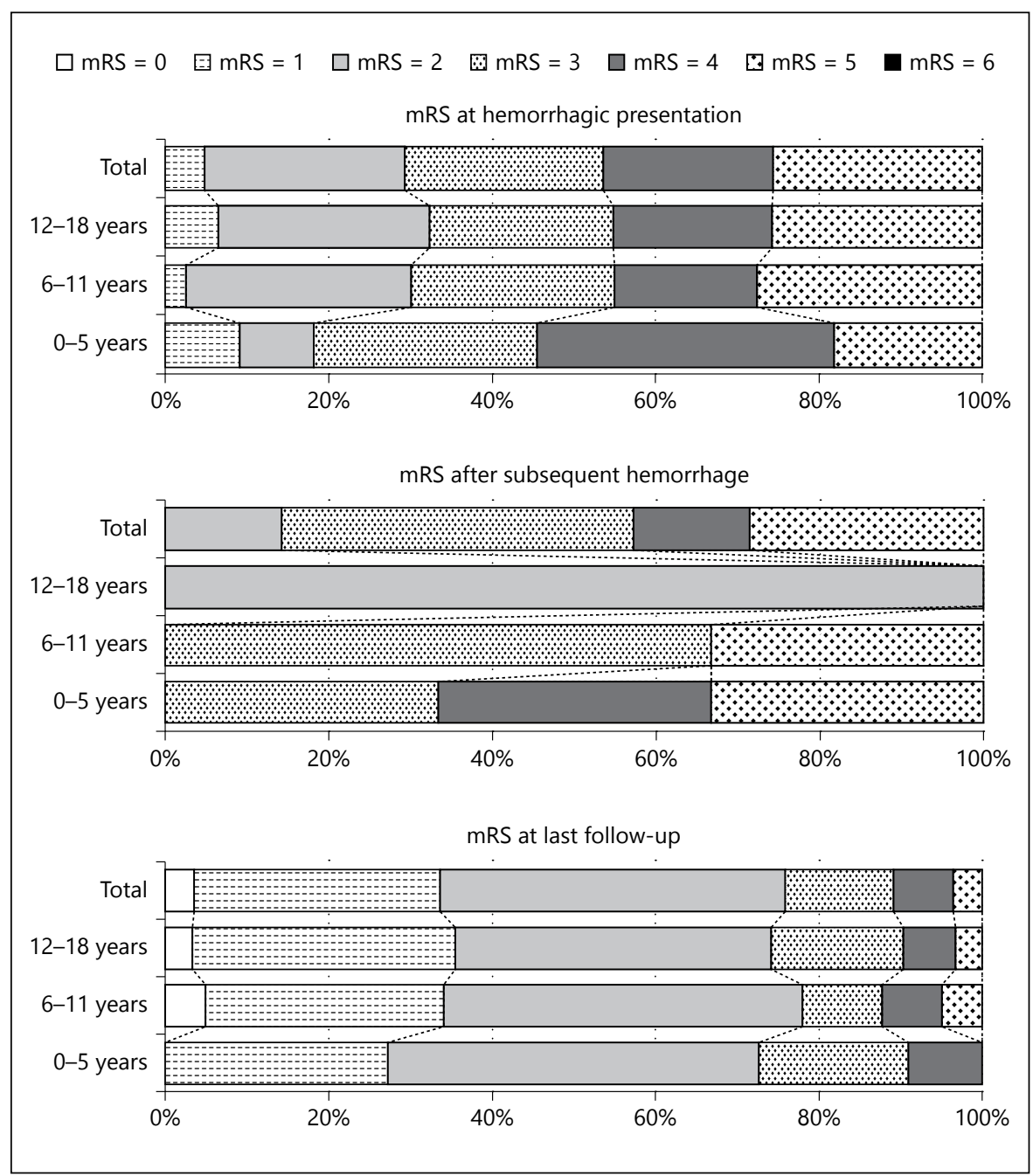

vere hemorrhage events occurred in children with severe hemorrhage history $(7 \%, 3 / 41)$, although the present data did not identify a higher risk of future severe hemorrhage in children with severe hemorrhage history (OR 17.0; 95\% CI 0.86-337.2). Subsequent hemorrhages tended to increase morbidity (median mRS 3 , mean mRS $3.6 \pm 1.1$ ) compared with the hemorrhagic presentation (median mRS 3, mean mRS $3.4 \pm 1.6$ ), while the difference was not significant ( $p=0.69$; Table 2$)$.

The mRS score at treatment-free follow-up was determined before bAVM treatment or at last follow-up for those who were under conservative observation (Table 2; Fig. 2). Twenty children (20/83, 24\%) with ruptured bAVM had an mRS score $\geq 3$ at follow-up. Neurological function at follow-up (median mRS 2, mean mRS $2.0 \pm 1.2$ ) was improved over time compared with $\mathrm{mRS}$ at hemorrhagic presentation (median mRS 3, mean mRS $3.4 \pm 1.6)$. The $\mathrm{mRS}$ at follow-up was positively correlated with $\mathrm{mRS}$ at presentation $(R=0.58, p<$ $0.001)$.

\section{Morphologic Features Associated with Severe Hemorrhage}

In univariate analysis, severe hemorrhage ( $\mathrm{mRS}>3$ or requiring emergency hematoma evacuation after hemorrhagic presentation or subsequent hemorrhage) occurred at a significantly higher frequency in bAVMs in periventricular location $(p=0.003)$ and non-temporal lobe $(p=$ 0.03 ), whereas we did not find any association between other anatomical locations and severe hemorrhage. There was a trend for more severe hemorrhage to occur in bAVMs with long draining vein $(p=0.06$; Table 1$)$.

Multivariable logistic analyses were performed to define independent risk factors for severe bAVM hemor- 
Table 2. Hemorrhage risk and post-hemorrhage clinical outcome in children with untreated brain arteriovenous malformation*

\begin{tabular}{|c|c|c|c|c|c|c|}
\hline & \multirow{2}{*}{$\begin{array}{l}\text { Hemorrhagic } \\
\text { presentation }\end{array}$} & \multicolumn{4}{|l|}{ Subsequent hemorrhage } & \multirow{2}{*}{$\begin{array}{l}\text { At last } \\
\text { treatment-free } \\
\text { follow-up }\end{array}$} \\
\hline & & $\begin{array}{l}\text { ruptured presentation } \\
(n=82)\end{array}$ & $\begin{array}{l}\text { unruptured presentation } \\
(n=52)\end{array}$ & $\begin{array}{l}\text { overall } \\
(n=134)\end{array}$ & $\begin{array}{l}\text { conservative } \\
\text { observation } \\
(n=21)\end{array}$ & \\
\hline $\begin{array}{l}\text { No. of patients with hemorrhage } \\
\text { mRS }\end{array}$ & 82 & $6(7.3)$ & $1(1.9)$ & $7(5.2)$ & $1(4.8)$ & 83 \\
\hline Mean \pm SD & $3.4 \pm 1.6$ & $3.8 \pm 1.0$ & $2 \pm 0$ & $3.6 \pm 1.1$ & $5 \pm 0$ & $2.0 \pm 1.2$ \\
\hline Median & 3 & 3.5 & 1 & 3 & 5 & 2 \\
\hline 0 & $0(0)$ & $0(0)$ & $0(0)$ & $0(0)$ & $0(0)$ & $3(3.6)$ \\
\hline 1 & $4(4.9)$ & $0(0)$ & $0(0)$ & $0(0)$ & $0(0)$ & $25(30.1)$ \\
\hline 2 & $20(24.4)$ & $0(0)$ & $1(100)$ & $1(14.3)$ & $0(0)$ & $35(42.2)$ \\
\hline 3 & $20(24.4)$ & $3(50)$ & $0(0)$ & $3(42.8)$ & $0(0)$ & $11(13.3)$ \\
\hline 4 & $17(20.7)$ & $1(16.7)$ & $0(0)$ & $1(14.3)$ & $0(0)$ & $6(7.2)$ \\
\hline 5 & $21(25.6)$ & $2(33.3)$ & $0(0)$ & $2(28.6)$ & $1(100)$ & $3(3.6)$ \\
\hline Emergent hematoma evacuation & $23(28)$ & $0(0)$ & $0(0)$ & $0(0)$ & $0(0)$ & $23(27.7)$ \\
\hline Severe hemorrhage & $41(50)$ & $3(50)$ & $0(0)$ & $3(42.9)$ & $1(100)$ & $41(49.4)$ \\
\hline Treatment-free follow-up & & & & & & \\
\hline (patient-years) & - & 88.5 & 198.4 & 286.9 & 189.5 & - \\
\hline Annual rupture rate, $\%$ & - & 7.9 & 0.5 & 2.8 & 0.5 & - \\
\hline Annual severe hemorrhage rate, $\%$ & - & 3.3 & 0 & 1.0 & 0.5 & - \\
\hline
\end{tabular}

Table 3. OR of demographic and morphologic features in predicting arteriovenous malformation severe hemorrhage in children*

\begin{tabular}{|c|c|c|c|}
\hline & Univariate & Model 1 & Model 2 \\
\hline Periventricular location & $3.31(1.46-7.51)$ & $3.89(1.64-9.27)$ & $3.95(1.44-10.84)$ \\
\hline Non-temporal lobe location & $2.61(1.08-6.27)$ & $3.19(1.25-8.15)$ & $2.89(1.09-7.70)$ \\
\hline Maximal AVM size, $\mathrm{mm}$ & $1.00(0.98-1.02)$ & - & $0.99(0.97-1.02)$ \\
\hline Age at diagnosis, years & $0.93(0.84-1.02)$ & - & $0.97(0.87-1.09)$ \\
\hline Gender, male & $0.77(0.37-1.63)$ & - & $0.69(0.29-1.64)$ \\
\hline Posterior fossa location & $1.39(0.32-6.11)$ & - & $1.43(0.26-7.94)$ \\
\hline Eloquence & $0.84(0.40-1.75)$ & - & $0.56(0.23-1.36)$ \\
\hline Exclusively deep venous drainage & $1.82(0.59-5.64)$ & - & $2.23(0.53-9.35)$ \\
\hline Associated aneurysm & $1.30(0.41-4.14)$ & - & $0.93(0.23-3.72)$ \\
\hline
\end{tabular}

* Table entries are OR (95\% CI); boldface indicates statistical significance. Model 1, logistic regression model with a forward stepwise regression procedure; model 2 , adjusted model with main effects.

rhage using 2 models: a forward stepwise model to test all variables listed in Table 3, excluding interactions between variables and a full model with all the variables entered, adjusting relative risks. In both of them, we identified periventricular location, non-temporal location, and long draining vein as predictors for severe hemorrhage. Age, gender, deep location, eloquent location, posterior fossa, exclusively deep venous drainage, and associated aneurysm did not have an effect in these pediatric patients.

Along with the multivariable regression analysis, we used a nomogram to show the risk stratification for severe hemorrhage based on the morphology of bAVM (Fig. 3a). For an individual patient, positive factors contributed to points and a total point corresponded to his probability of experiencing severe hemorrhage from an untreated bAVM. The calibration curve suggested that the nomogram was relatively well calibrated with minor discrepancies between observed and predicted probabilities (Fig. 3b). C-statistic of 0.74 (95\% CI 0.64-0.83) and 0.72 after bootstrap correction indicated good model discriminative ability. 


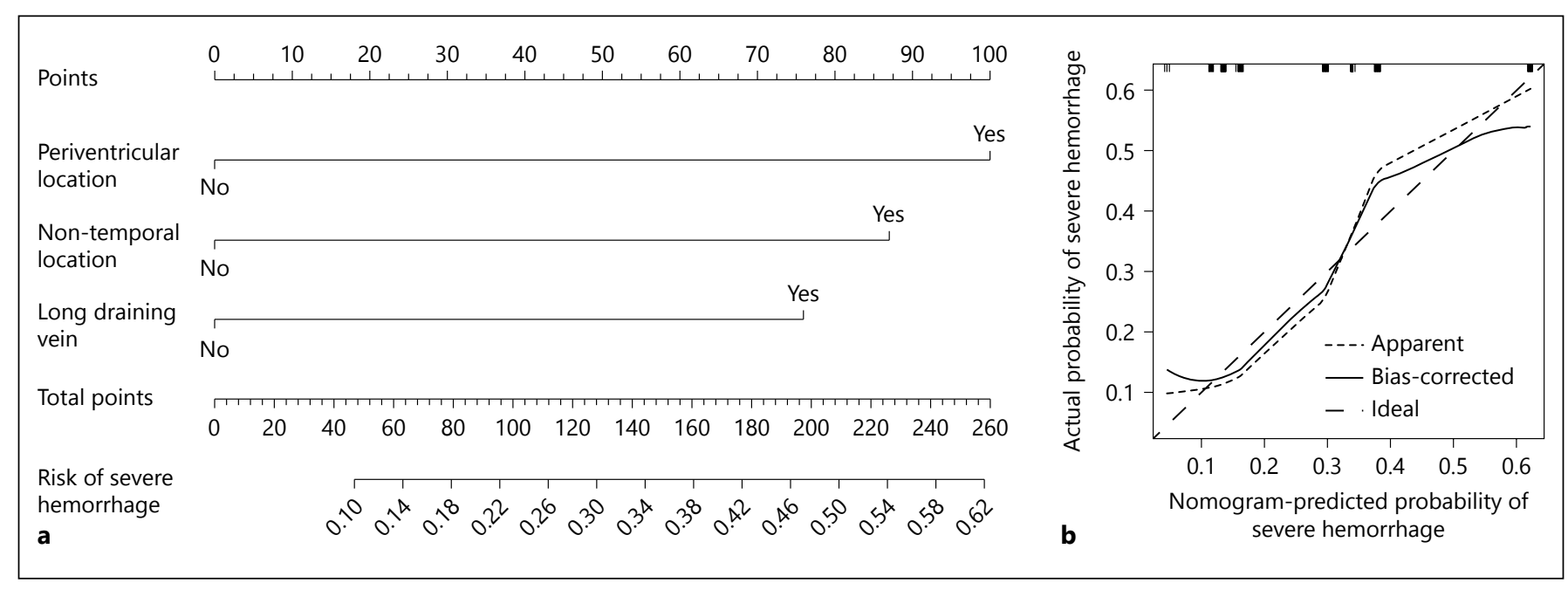

Fig. 3. Severe hemorrhage risk stratification. Nomogram for severe hemorrhage in children with untreated bAVM (a). To estimate the probability of severe hemorrhage for a specific patient, review his bAVM morphologic features listed in nomogram and then draw a vertical line through the feature status toward the Points axis to acquire the respective scores associated with each individual feature. The sum of 3 scores corresponds to a total score on the Total Points axis, a vertical line through which the Risk of Severe Hemorrhage axis will intersect at the predicted probability of severe hemorrhage. The predicted probability might be applicable to

\section{Discussion}

bAVMs are described as the underlying cause of a majority of childhood intracranial hemorrhage, while the ARUBA trial comparing bAVM natural history risk and treatment risk did not include pediatric patients [1]. Recent studies suggest that bAVM rupture may be more benign than previously assumed, with a lower morbidity than intracranial hemorrhage from other causes $[1,3,4]$. Therefore, to understand the morbidity of spontaneous hemorrhage and to predict the risk of severe hemorrhage might be helpful for weighing risks from untreated bAVM and invasive treatment. In our pediatric cohort, we assessed the neurological function after the first and recurrent hemorrhages during treatment-free follow-up.

\section{Morbidity of Pediatric bAVM Hemorrhage}

Hospital- or population-based cohort studies revealed an overall case fatality of $11-29 \%$ and severe disability rate of $14-38 \%$ after bAVM hemorrhage in adults [3, 17]. In our pediatric cases, $42-46 \%$ of children were severely disabled after hemorrhagic presentation or subsequent hemorrhage, which is congruent with previous data of treated pediatric bAVM series $(32-50 \%)[5,6]$. While hemorrhagic presentation and subsequent hemorrhage during an average 2-year follow-up after diagnosis in children with untreated bAVM. Calibration curve for internal validation of the nomogram (b). The rug plots across the top of the graph show the distribution and quantities of data used to fit the model. The dashed line represents the performance of ideal nomogram where predicted probability perfectly corresponds with observed probability. The dotted line shows the apparent accuracy of our nomogram without correction for overfit. The solid line shows the bootstrap-corrected performance of our nomogram.

some previous reports on bAVM patients of all ages suggested a lower rate of severe disability after incident hemorrhage (3-45\%), however, a longer delay between bAVM rupture and neurological assessment might be one of the factors for the prevalence of lower rates in those studies (Table 4) $[3,18]$. Additionally, we only found a trend that showed that a mild higher mRS score was observed after hemorrhage in younger children (Fig. 2; online suppl. material). Therefore, further observation is still required to determine whether the morbidity of bAVM rupture is correlated with age and whether it is different between children and adults.

During treatment-free follow-up, the annual rate of subsequent severe hemorrhage (overall 1.0\%, conservative observation subgroup $0.5 \%$ ) and annual rupture rate $(2.8 \%)$ in this pediatric cohort (Table 2) were similar to that quoted in other study of patients of all ages (severe hemorrhage rate of $1.4 \%$ and rupture rate of $2-4 \%$ ) [19$21]$. However, the cumulative rupture risk and morbidity could be high, given the long life expectancy of pediatric patients. Moreover, our follow-up data suggest that children with a severe hemorrhage history might be at higher risk for poor outcome after new hemorrhage. All the subsequent severe hemorrhage occurred in children with a 
Table 4. Clinical outcome of patients with untreated brain arteriovenous malformation after presenting and subsequent hemorrhages from different studies

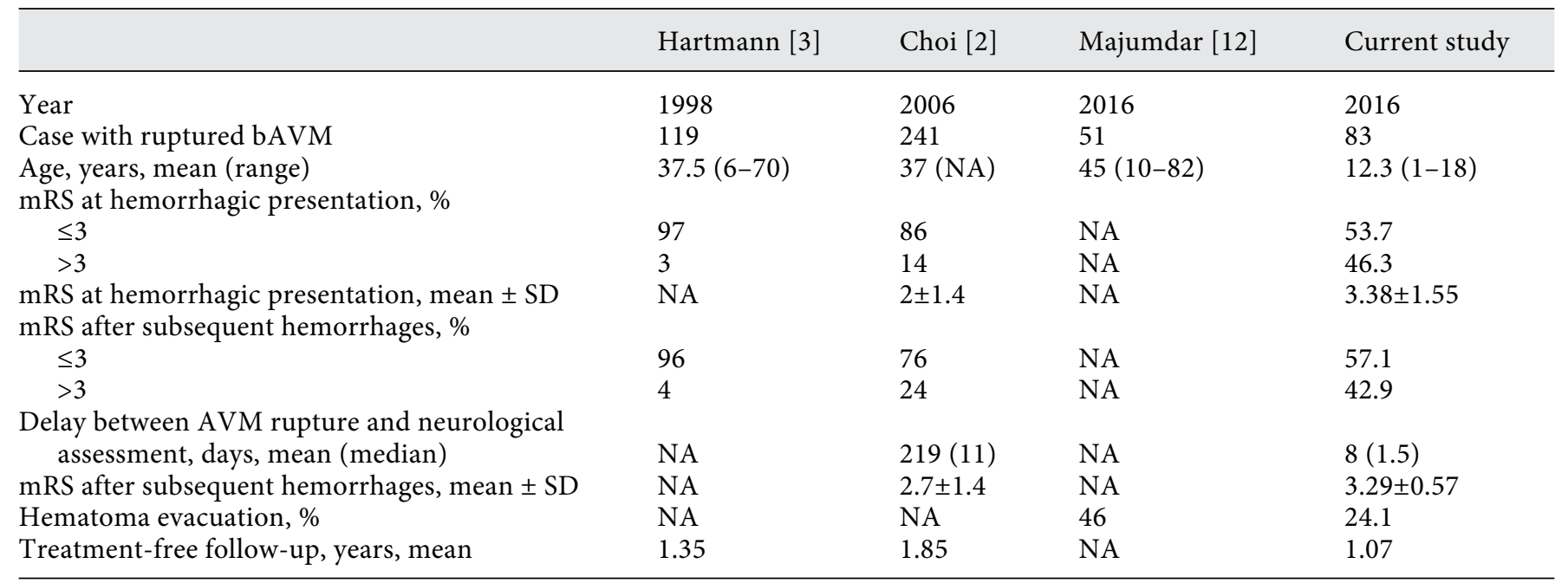

NA, not applicable.

severe hemorrhagic presentation during a short-term follow-up, while the treatment-free follow-up time was similar between children with or without severe hemorrhagic history (log-rank, $p=0.54$, even shorter in children with severe hemorrhagic history, mean 1.3 vs. 2.5 years) (Table 1; online suppl. material), suggesting that the risk of subsequent severe hemorrhage in children with severe hemorrhagic history might not be overestimated. Thus, pediatric bAVM patients with a severe hemorrhage history should be recommended to seek treatment for obliteration of the lesion. It should be noted that around 50\% of pediatric bAVMs are not diagnosed until they rupture, and we observed subsequent hemorrhage only in one unruptured bAVM. Therefore, the morbidity of severe hemorrhage in unruptured bAVM still needs more studies.

Another important functional outcome measurement is the disability at longer-term follow-up. Although we found that $76 \%$ of children with ruptured bAVM were independent ( $\mathrm{mRS}<3$ ) during follow-up $(55-95 \%$ reported in patients of all ages) $[3,4,18]$, this should be interpreted with caution. Eighty-four percent of our cases underwent interventional treatment for the lesion, while the intervals between index event and treatment were individualized. In the presumably lower-risk subgroup of 21 conservatively managed patients, $73 \%$ of children with ruptured bAVM (8/11) were independent during followup. Interventional treatment might prevent a devastating outcome in some cases but censor follow-up of others in rehabilitation. However, a majority of patients with rup- tured bAVM would be recommended for intervention considering a higher future hemorrhage risk. Therefore, it is relatively difficult to describe the long-term functional outcome after bAVM hemorrhage in a natural course.

\section{Predictive Features for Severe Hemorrhage in \\ Pediatric bAVM}

Severe morbidity of bAVM hemorrhage could be influenced by hemorrhage risk and hemorrhage location and it involved brain function. Previous studies on the functional outcome of bAVM hemorrhage were mainly focused on ruptured bAVM, which excluded the effect of different hemorrhage risk among bAVM of various features (Table 4). Therefore, predictive factors identified in these studies might not be associated with severe hemorrhage in all bAVM patients. For instance, in ruptured bAVM, eloquent location is associated with severe hemorrhage. However, it is not a predictor for higher hemorrhage risk [22, 23]. In addition, the eloquence includes functional cortex, deep brain, and brain stem, while some important subcortical tracts are outside the scope of the definition. Compared with the cortex, tract injury seems to result in a more long-term neurological deficit, where the functional reorganization might be slow [24].

In this study, we identified periventricular nidus location, non-temporal lobe location, and long draining vein as independent predictors for severe hemorrhage. The periventricular brain includes not only a vital structure in 
the deep brain and brain stem but also important subcortical tracts, which are not classified as either deep brain or eloquent region. Our previous data indicated that $87 \%$ of periventricular ruptured bAVMs had parenchymatous hemorrhage with or without intraventricular extension, which could lead to the disruption of important nucleus and/or tracts [8], and parenchymal hemorrhage is associated with increased morbidity in bAVM $[3,18]$. These features might explain the reason for higher risk for severe hemorrhage of periventricular bAVM.

Interestingly, temporal lobe location was identified as a protective feature for unfavorable outcomes after hemorrhage in our pediatric series. The anatomical characteristics of temporal AVM might be associated with a better outcome. Most temporal lobe is not considered eloquent region and can even be surgically resected without significant neurological deficits [25]. In addition, most temporal AVM were on the lateral convexity and inferior base of temporal lobe [26], where the eloquent regions might not be involved. Moreover, language cortex reorganization occurred in more bAVMs involving the Wernicke area of the temporal lobe than those involving the Broca area of the frontal lobe [27].

Long draining vein was also suggested as a risk factor for causing severe hemorrhage. Our recent study indicated that restricted venous outflow was associated with higher risk of bAVM future hemorrhage [28]. Therefore, it might be feasible for future studies to test more features suggesting bAVM hemodynamics.

With this short-term treatment-free follow-up and limited occurrence of subsequent hemorrhage, we were not able to perform a Cox regression for the subsequent severe hemorrhage. Thus, predictive features identified in this study were more likely to be associated with severe hemorrhagic presentation. However, the higher risk for subsequent severe hemorrhage after a severe hemorrhagic presentation suggests that predicting severe hemorrhagic presentation might also be helpful. With the aforementioned morphologic features, we proposed a risk stratification model for severe hemorrhage in pediatric untreated bAVM. Although internal validation indicates an overall good performance, its predictive accuracy should be evaluated in an independent cohort. Additionally, further analysis suggested high specificity $(0.90)$ and low sensitivity $(0.32)$ for this nomogram. Therefore, future studies with a larger sample size and more predictive features (i.e., clinical, hemodynamic, and more detailed morphology) are required to improve the risk-stratification system.

This study was limited by its single-institutional population, potential bias, and follow-up time. The study pop- ulation was based on the children who have presented themselves to medical attention. However, some patients with very severe intracranial hemorrhage might not be able to undergo the diagnostic radiological examination or refer to our institute to make a diagnosis, and therefore, were not included due to the indefinite diagnosis or referral bias. Additionally, the interventional treated bAVM might be different from those with conservative management (online suppl. material). Therefore, the hemorrhage risk and morbidity of bAVM patients with conservative management were described separately. Considering these potential biases, the present results should be interpreted with caution. The occurrence of subsequent hemorrhage during short-term treatment-free follow-up also limited further survival analysis. Future studies of larger sample size, with more accurate noninvasive diagnostic tools and longer follow-up could expand this topic.

\section{Conclusions}

Careful evaluation of bAVM morbidity and morphology might be helpful for weighing the risks of untreated bAVM in pediatric patients.

\section{Acknowledgments}

The authors would like to thank Dr. Gu Wei-Bin of the Department of Neuroradiology at Beijing Tiantan Hospital for his assistance with the image analysis, and Dr. Yuan Lian-Xiong at the Sun Yat-Sen University and Jeffrey Nelson at the University of California San Francisco for their assistance with the data analysis.

\section{Funding}

This project was supported by the Ministry of Science and Technology of China, National Key Technology Research and Development Program (2015BAI12B04, 2013BAI09B03, 2012CB720704); Beijing Institute for Brain Disorders grant (BIBD-PXM2013_014226_07_000084); and National Natural Science Foundation of China (H0906 81271313 and H0906 81571110 to Y.Z.); and National Institutes of Health (R01 NS034949 to H.K., R01 NS027713, R01 HL122774 and R21 NS083788 to H.S.); and Health Industry Special Scientific Research Project (No. 201402019 to J.M.); and China Scholarship Council (No. 201508110252 to L.M.) and Michael Ryan Zodda Foundation.

\section{Disclosure Statement}

The authors have no conflicts of interest to declare. 


\section{References}

1 Fullerton HJ, Achrol AS, Johnston SC, McCulloch CE, Higashida RT, Lawton MT, Sidney S, Young WL; UCSF BAVM Study Project: Long-term hemorrhage risk in children versus adults with brain arteriovenous malformations. Stroke 2005;36:2099-2104.

2 Ding D, Yen CP, Starke RM, Xu Z, Sheehan JP: Effect of prior hemorrhage on intracranial arteriovenous malformation radiosurgery outcomes. Cerebrovasc Dis 2015;39:53-62.

3 Choi JH, Mast H, Sciacca RR, Hartmann A, Khaw AV, Mohr JP, Sacco RL, Stapf C: Clinical outcome after first and recurrent hemorrhage in patients with untreated brain arteriovenous malformation. Stroke 2006;37:12431247.

4 Hartmann A, Mast H, Mohr JP, Koennecke HC, Osipov A, Pile-Spellman J, Duong DH, Young WL: Morbidity of intracranial hemorrhage in patients with cerebral arteriovenous malformation. Stroke 1998;29:931-934.

5 Blauwblomme T, Bourgeois $\mathrm{M}$, Meyer $\mathrm{P}$, Puget S, Di Rocco F, Boddaert N, Zerah M, Brunelle F, Rose CS, Naggara O: Long-term outcome of 106 consecutive pediatric ruptured brain arteriovenous malformations after combined treatment. Stroke 2014;45: 1664-1671.

6 Gross BA, Storey A, Orbach DB, Scott RM, Smith ER: Microsurgical treatment of arteriovenous malformations in pediatric patients: the Boston children's hospital experience. J Neurosurg Pediatr 2015;15:71-77.

7 Abla AA, Nelson J, Rutledge WC, Young WL, Kim H, Lawton MT: The natural history of AVM hemorrhage in the posterior fossa: comparison of hematoma volumes and neurological outcomes in patients with ruptured infra- and supratentorial AVMs. Neurosurg Focus 2014;37:E6.

8 Ma L, Huang Z, Chen XL, Ma J, Liu XJ, Wang $\mathrm{H}$, Ye X, Wang SL, Cao Y, Wang S, Zhao YL, Zhao JZ: Periventricular location as a risk factor for hemorrhage and severe clinical presentation in pediatric patients with untreated brain arteriovenous malformations. AJNR Am J Neuroradiol 2015;36:1550-1557.

9 Bigi S, Fischer U, Wehrli E, Mattle HP, Boltshauser E, Burki S, Jeannet PY, Fluss J, Weber P, Nedeltchev K, El-Koussy M, Steinlin M, Arnold M: Acute ischemic stroke in children versus young adults. Ann Neurol 2011;70: 245-254.
10 Maas MB, Francis BA, Sangha RS, Lizza BD, Liotta EM, Naidech AM: Refining prognosis for intracerebral hemorrhage by early reassessment. Cerebrovasc Dis 2017;43:110-116.

11 Atkinson RP, Awad IA, Batjer HH, Dowd CF, Furlan A, Giannotta SL, Gomez CR, Gress D, Hademenos G, Halbach V, Hemphill JC, Higashida RT, Hopkins LN, Horowitz MB, Johnston SC, Lawton MW, McDermott MW, Malek AM, Mohr JP, Qureshi AI, Riina H, Smith WS, Pile-Spellman J, Spetzler RF, Tomsick TA, Young WL: Reporting terminology for brain arteriovenous malformation clinical and radiographic features for use in clinical trials. Stroke 2001;32:1430-1442.

12 Koo HW, Jo KI, Yeon JY, Kim KH, Jeon P, Kim JS, Hong SC, Shin HJ, Lee JI: Clinical features of superficially located brain arteriovenous malformations with transdural arterial communication. Cerebrovasc Dis 2016;41: 204-210.

13 Meder JF, Oppenheim C, Blustajn J, Nataf F, Merienne L, Lefkoupolos D, Laurent A, Merland JJ, Schlienger M, Fredy D: Cerebral arteriovenous malformations: the value of radiologic parameters in predicting response to radiosurgery. AJNR Am J Neuroradiol 1997; 18:1473-1483.

14 Shankar JJ, Menezes RJ, Pohlmann-Eden B, Wallace C, terBrugge K, Krings T: Angioarchitecture of brain AVM determines the presentation with seizures: proposed scoring system. AJNR Am J Neuroradiol 2013;34:10281034.

15 Stein KP, Wanke I, Forsting M, Oezkan N, Huetter BO, Sandalcioglu IE, Sure U: Associated aneurysms in infratentorial arteriovenous malformations: role of aneurysm size and comparison with supratentorial lesions. Cerebrovasc Dis 2016;41:219-225.

16 Stein KP, Wanke I, Forsting M, Zhu Y, Moldovan AS, Dammann P, Sandalcioglu IE, Sure $\mathrm{U}$ : Associated aneurysms in supratentorial arteriovenous malformations: impact of aneurysm size on haemorrhage. Cerebrovasc Dis 2015;39:122-129.

17 van Beijnum J, Lovelock CE, Cordonnier C, Rothwell PM, Klijn CJ, Al-Shahi Salman R; SIVMS Steering Committee and the Oxford Vascular Study: Outcome after spontaneous and arteriovenous malformation-related intracerebral haemorrhage: population-based studies. Brain 2009;132(pt 2):537-543.

18 Majumdar M, Tan LA, Chen M: Critical assessment of the morbidity associated with ruptured cerebral arteriovenous malformations. J Neurointerv Surg 2016;8:163-167.
19 Crawford PM, West CR, Chadwick DW, Shaw MD: Arteriovenous malformations of the brain: natural history in unoperated patients. J Neurol Neurosurg Psychiatry 1986; 49:1-10.

20 Hernesniemi JA, Dashti R, Juvela S, Vaart K, Niemela M, Laakso A: Natural history of brain arteriovenous malformations: a longterm follow-up study of risk of hemorrhage in 238 patients. Neurosurgery 2008;63:823-829; discussion 829-831.

21 Yang W, Anderson-Keightly H, Westbroek EM, Caplan JM, Rong X, Hung AL, Colby GP, Coon AL, Tamargo RJ, Huang J, Ahn ES: Long-term hemorrhagic risk in pediatric patients with arteriovenous malformations. J Neurosurg Pediatr 2016;18:329-338.

22 Kim H, Al-Shahi Salman R, McCulloch CE, Stapf C, Young WL;MARS Coinvestigators : Untreated brain arteriovenous malformation: patient-level meta-analysis of hemorrhage predictors. Neurology 2014;83:590-597.

23 Gross BA, Du R: Natural history of cerebral arteriovenous malformations: a meta-analysis. J Neurosurg 2013;118:437-443.

24 Lin F, Zhao B, Wu J, Wang L, Jin Z, Cao Y, Wang S: Risk factors for worsened muscle strength after the surgical treatment of arteriovenous malformations of the eloquent motor area. J Neurosurg 2016;125:289-298.

25 Gabarros A, Young WL, McDermott MW, Lawton MT: Language and motor mapping during resection of brain arteriovenous malformations: indications, feasibility, and utility. Neurosurgery 2011;68:744-752.

26 Gabarros Canals A, Rodriguez-Hernandez A, Young WL, Lawton MT; UCSF Brain AVM Study Project: Temporal lobe arteriovenous malformations: anatomical subtypes, surgical strategy, and outcomes. J Neurosurg 2013; 119:616-628.

27 Deng X, Zhang Y, Xu L, Wang B, Wang S, Wu J, Zhang D, Wang R, Wang J, Zhao J: Comparison of language cortex reorganization patterns between cerebral arteriovenous malformations and gliomas: a functional MRI study. J Neurosurg 2015;122:996-1003.

28 Ma L, Chen XL, Chen Y, Wu CX, Ma J, Zhao YL: Subsequent haemorrhage in children with untreated brain arteriovenous malformation: higher risk with unbalanced inflow and outflow angioarchitecture. Eur Radiol 2016, Epub ahead of print. 